\title{
La investigación internacional de nuevos medicamentos: una valoración desde la justicia global*
}

\author{
International research of new drugs: \\ an evaluation from the global justice
}

\section{A pesquisa internacional de novos remédios: uma avaliação a partir da justiça global}

\author{
Fecha de recepción: 13 de enero de 2016 \\ Fecha de evaluación: 11 de abril de 2016 \\ Fecha de aceptación: 15 de abril de 2016 \\ Disponible en línea: 18 de abril de 2016 \\ Ricardo Páez Moreno** \\ DoI: http://dx.doi.org/10.18359/rlbi.1689.
}

Cómo citar:

Páez Moreno, R. (2016). La investigación internacional de nuevos medicamentos: una valoración desde la justicia global. Revista Latinoamericana de Bioética, 16(2), 188-213. DOI: http://dx.doi.org/10.18359/rlbi.1689.

Artículo de reflexión.

* Licenciado en Medicina, de la Universidad de La Salle, México; magíster en Bioética Universidad Pontificia Comillas, Madrid y doctor en Bioética de la Universidad Nacional Autónoma de México (UNAM). Profesor y tutor del Programa Universitario en Bioética, de la UNAM. Correo: ricardomsps@gmail.com. ORCID: http://orcid.org/0000-0001-7047-9983. Ciudad de México, México. 


\title{
Resumen
}

La investigación internacional de nuevos medicamentos patrocinada por la industria farmacéutica muestra una serie de conflictos de interés, que ocasionan muchas faltas a la justicia. Cuando la bioética los ha abordado desde la justicia de tipo nacionalista, ha pasado por alto muchas faltas. Por el contrario, algunos enfoques cosmopolitas, sobre todo de tipo contextual, ofrecen una mirada crítica que desenmascara las grandes desigualdades globales. Además, su abordaje a partir de las capacidades y necesidades de los involucrados y teniendo como horizonte el bienestar, ofrece guías mucho más concretas y accesibles a favor de acciones orientadas hacia la equidad.

Es un reto para la bioética reflexionar en modelos teóricos que ofrezcan, para las investigaciones internacionales, guías para evitar el abuso y favorecer una distribución de beneficios más equitativa. En este sentido, el objetivo de este artículo es presentar una reflexión sobre algunas importantes teorías de la justicia global y ciertos aspectos que pueden aplicarse, que orienten hacia una práctica más justa en la investigación internacional.

Palabras clave: justicia, investigaciónmédica, industria farmacéutica, bioética.

\begin{abstract}
International research of new medicines sponsored by the Pharmaceutical Industry creates a series of conflicts of interest, which result in many faults to justice. Whenever bioethics has approached this subject based on a nationalist approach to justice, many faults have been overlooked. By contrast, some cosmopolitan approaches, in particular, the contextual ones, offer a critical perspective, which unveils significant global inequalities. Moreover, they approach the subject based on the capacities and needs of those involved, while keeping well-being on the horizon, and therefore they offer more concrete and accessible guidelines for actions that will lead to equality.

It is a challenge for bioethics to reflect on theoretical models that will provide effective guidelines to prevent abuse and encourage a more equitable distribution of benefits. In this sense, the goal of this article is to provide a reflection on important theories of global justice and some areas where to be applied, to guide to a more just practice in international research.
\end{abstract}

Keyword: Justice, medical research, the pharmaceutical industry, bioethics.

\section{Resumo}

A pesquisa internacional de novos remédios patrocinada pela indústria farmacêutica mostra uma série de conflitos de interesse, que causam muitas faltas à justiça. Quando a bioética os abordou a partir da justiça de tipo nacionalista, tem negligenciado muitas falhas. Por outro lado, algumas abordagens cosmopolitas, especialmente sobre tudo de tipo contextuais, oferecem um olhar crítico que desmascara as grandes desigualdades globais. Além disso, sua abordagem a partir das capacidades e necessidades das pessoas envolvidas e tendo como alvo o bem-estar, oferece guias muito mais concretas e acessíveis em favor de ações orientadas para a equidade.

É um desafio para a bioética refletir sobre modelos teóricos que forneçam para as pesquisas internacionais, diretrizes para evitar o abuso e incentivar uma distribuição mais equitativa dos benefícios. Neste sentido, o objetivo deste artigo é apresentar uma reflexão sobre algumas teorias importantes da justiça global e determinados aspectos que podem ser aplicados, que orientem no sentido de uma prática mais justa na pesquisa internacional.

Palavras-chave: : justiça, pesquisa médica, indústria farmacêutica, bioética. 


\section{Introducción}

La investigación biomédica de nuevos tratamientos patrocinada por la industria farmacéutica es un fenómeno propio de la globalización, al realizarse simultáneamente en múltiples países y centros de investigación. Al combinarse las dinámicas de neutralidad en la investigación con la necesidad de ganancia económica desmedida, se ha mostrado una serie de conflictos de interés científicos y económicos, que requieren una reflexión bioética global (Páez Moreno, 2011). La justicia, que es la categoría bioética que más se pone en entredicho, es necesario pensarla a nivel global (Pogge, 2002). Sin embargo, no existe un único abordaje de justicia global y cada uno ofrece posturas distintas. La postura nacionalista que defiende la tesis "fuera del Estado no hay justicia” (Nagel, 2005) puede justificar el doble estándar entre países en las investigaciones internacionales. Pero pueden surgir requerimientos éticos desde otros abordajes, como los cosmopolitas, que identifican normas de justicia aplicables a todo el mundo por igual, sin hacer distinciones de países o regiones. Dentro de estos enfoques hay los de corte utilitarista, que se sustentan en el principio de mayor felicidad para el mayor número, y por lo mismo argumentan la necesidad de distribuir los beneficios con aquellos que están peor (Singer, 2003). O los que tienen que ver con el respeto a los derechos humanos, en concreto al deber de no dañar, puesto que la valoración moral de las personas y de sus conductas, de las reglas sociales y de los estados de las cosas debe basarse en principios que rijan a todos por igual (Pogge, 2005a). En estos últimos se basan agudos planteamientos de reforma de las prácticas de la industria farmacéutica.

Los enfoques cosmopolitas contextuales tienen mucho que decir a la investigación internacional. En un mundo donde el poderoso abusa del débil, las capacidades y necesidades son muy desiguales, $y$ en algunos sitios no hay un Estado que proteja a las mayorías, se requiere empoderar al necesitado y disciplinar al fuerte (O' Neill, 2000b). La justicia global tiene que ver con el bienestar de individuos y poblaciones, pero también con las causas que ocasionan desniveles profundos y duraderos que impiden el acceso al bienestar (Powers y Faden, 2013). Las capacidades en salud, entendidas como habilidad para la toma de decisiones y ayudar a otros e influir en las políticas e instituciones, requieren un trabajo conjunto para reducir las grandes diferencias (Ruger, 2010).

Ante los diversos hechos de injusticia acontecidos en la investigación biomédica internacional y a la luz de importantes teorías de la justicia global, este trabajo tiene como objetivo ofrecer algunos elementos que orienten hacia una práctica más justa en la investigación. Para ello, se presentan de manera sucinta algunas expresiones del conflicto de interés existente en la investigación internacional. Continúa una breve introducción al debate entre las teorías estatistas o nacionalistas y las cosmopolitas. En 
seguida se desarrollan cinco enfoques de justicia de tipo cosmopolita: la propuesta utilitarista de Peter Singer sobre la noción de igualdad de todas las personas en necesidad; la postura basada en los derechos humanos de Thomas Pogge; el enfoque de justicia global partiendo de las posibilidades de acción según Onora O' Neill; el abordaje de justicia global de Madison Powers y Ruth Faden; y finalmente el paradigma de las capacidades en salud de Jennifer Ruger. Por último, a la luz del marco teórico presentado, se hace un balance y se presentan cinco situaciones en las que puede incidirse para una investigación internacional más equitativa.

\section{Conflictos de interés en la investigación biomédica global}

La investigación biomédica a nivel global para descubrir nuevos medicamentos para el tratamiento de diversas enfermedades es llevada a cabo por la industria farmacéutica cada vez más, hecho que desplaza a la investigación patrocinada por los Estados. Se organiza y coordina desde grandes empresas localizadas sobre todo en países de alto ingreso, tales como Estados Unidos, Japón o los de la Comunidad Europea. Y se llevan a cabo en diversos centros hospitalarios en todo el mundo, sobre todo en países de mediano o bajo ingreso, muchas veces a expensas de la medicina pública que tiene gran cantidad de pacientes y mayores posibilidades de investigación, utilizando la planta investigadora local (Páez Moreno, 2015).
La investigación internacional es un fenómeno que forma parte de la globalización económica y es necesario entenderla como parte de este fenómeno mundial. El mundo de capitales e inversiones, del cual forma parte importante la investigación farmacéutica, ha cobrado enormes dimensiones a partir de la globalización, apareado de varios problemas éticos, muchos de ellos producto de un conflicto de intereses científico y económico. Uno de ellos es la neutralidad científica que se ha menoscabado a expensas de la ganancia económica, aplazando e incluso negando resultados no convenientes, publicando éxitos no tan relevantes y en ocasiones con el nombre de grandes investigadores que fungen como autores fantasma (Pignarre, 2005; Bekelman, Mphil y Gross 2003; Páez Moreno, 2011).

Aunado a lo anterior, se ha demostrado el descuido en investigar en enfermedades que afectan a la mayoría de la población mundial, concentrándose en aquellas que ofrecen un beneficio económico debido a que afectan a pacientes con capacidad de pago de los nuevos medicamentos (Pogge, 2002). Hasta hace poco se privilegiaba la investigación en la cura de enfermedades de países de alto ingreso (Páez Moreno, 2009); actualmente se está desviando la atención a investigar tratamientos para enfermedades no comunicables (Bollyky, 2013), que parecen ser las más rentables debido a la transición epidemiológica sufrida en varios países de mediano ingreso, tales como México. Pero se descuida investigar 
en aquellos padecimientos que afectan a quienes soportan la mayor carga de la enfermedad y que se curarían con poco esfuerzo. El dato crudo que más revela la injusticia global en esta área es que la investigación global está marcada por el cociente 10/90, es decir, que solo el 10 $\%$ de la población mundial se beneficia del 90\% de los recursos destinados a la investigación, y el 90\% restante tiene que conformarse con el 10\% de los recursos (Michaud y Murray, 1996; World Medical Association, 2015).

En tercer lugar y sin pretender con ello agotar el elenco de conflictos de interés, es necesario tomar en cuenta el fenómeno de las patentes, las cuales se adquieren por parte de la Food and Drug Administration de los Estados Unidos por un periodo de 20 años a partir del momento cuando es aprobado el protocolo de investigación de una nueva molécula. Durante la comercialización del tratamiento exitoso, el alto precio fijado es la manera de obtener enormes ganancias sin competencia. Esto supone para la industria farmacéutica patrocinadora una carrera contra el tiempo en orden a conseguir la patente antes que la competencia, reclutando pacientes en todo el mundo y utilizando varios centros de investigación para obtener más rápido los resultados pretendidos, $\mathrm{O}$ tener que recurrir a diversas estrategias para obtener extensiones de las patentes, por ejemplo a través de leves modificaciones a las moléculas originales, lo que se ha llamado evergreening strategies (Vernaz, 2013).
La ética de la investigación exige, por lo tanto, una reflexión bioética global. La justicia, que es la más vulnerada en este tipo de investigaciones, tiene que ser pensada desde lo global, puesto que las investigaciones internacionales son un fenómeno propio de la globalización, cuyo comportamiento obedece a las reglas del mercado globales. No bastaría un abordaje desde la justicia retributiva propia de las obligaciones entre contratantes, o la justicia distributiva pensada desde principios imparciales de repartición de bienes, pero que poco toman en cuenta las tan disímbolas realidades que pueden afectar a los interesados. Se ha señalado que la mayoría de la literatura en bioética sobre el tema parte de estos abordajes, y se da así origen a pronunciamientos minimalistas (Macklin, 2004; London, 2005). En el caso de la justicia internacional o global, los contextos entre los distintos países son tan diversos y la diferencia de poder es tan desventajosa, que requiere una manera alternativa de pensar la justicia en vistas a que se evite lo más posible el conflicto de interés.

\section{Teorías globales de justicia}

Como parte de la reflexión sobre lo justo a nivel global, existe una acalorada discusión sobre las fronteras que debe abarcar la justicia, es decir, si esta es materia de cada país o región, o si pertenece a un ámbito cosmopolita y supranacional. Se trata de un desacuerdo filosófico sustancial sobre si hay obligaciones internacionales de justicia para reducir 
tales inequidades y para proteger mejor los derechos a la salud de aquellos cuyas sociedades no los protegen tanto como debieran. $^{2}$

En el ámbito académico anglosajón hay un largo e irreconciliable debate entre nacionalistas y cosmopolitas. Los nacionalistas dicen que solo puede velarse por la justicia de aquellos que están bajo una denominación nacional. Existen nacionalistas fuertes y moderados; los primeros aducen que no hay normas robustas de justicia social que tengan aplicación más allá del Estado-nación, y los segundos argumentan que mientras pueda haber ciertas normas de justicia social que tengan aplicación más allá del Estado-nación, estas no son tan demandantes como las variadas normas igualitarias que se aplican domésticamente. Los nacionalistas fuertes concluyen que las normas del mercado son las únicas relevantes para sostener el orden económico global. Defienden la visión del mercado libre como moralmente adecuada fuera del contexto doméstico, no importando sus efectos distributivos transnacionales, siempre y cuando las transacciones no sean producto del fraude o la coerción entre las partes. No hay límite en las inequidades permisibles en cuanto a tener que modificar las políticas de las mayores instituciones que regulan el orden económico global. No hay deberes de las naciones o de las corporaciones multinacionales en cuanto a abstenerse

1 Para una visión complexiva sobre el debate relacionado con la justicia global, ver: Brooks (2008). de tomar ventaja de las grandes diferencias en el poder de embargo de las transacciones económicas globales. ${ }^{3}$

Varios de los conflictos de interés de la industria farmacéutica quedan justificados con las teorías nacionalistas. El lema de los nacionalistas fuertes es "extra republicam nulla justitia", marcando un doble estándar para evaluar lo justo, dependiendo de si acontece dentro del propio Estado o fuera de este. En otras palabras, es la justificación ideal para el doble estándar en la investigación internacional. Dentro de la propia nación se hace justicia, no así fuera de ella. Thomas Nagel (2005) es un autor prototipo de este tipo de pensamiento. Desde este enfoque se justifican las investigaciones internacionales siempre y cuando no sean fruto de la trampa o la coacción. Es la base para justificar un doble estándar entre los países de alto ingreso y los demás.

Una postura más moderada es la de John Rawls (2001), quien no deja de establecer un doble rasero para evaluar lo justo. La misión asignada a las partes en la posición original internacional difiere considerablemente de la doméstica; su función no es, como se podría esperar, lograr un acuerdo sobre los criterios públicos para la valoración, diseño y reforma del orden global institucional, sino acordar un conjunto de normas

Existen también los defensores de las teorías híbridas, en cuanto comparten de diversas maneras elementos de nacionalistas y cosmopolitas. 
de buena conducta que los pueblos en cooperación deben obedecer. A los Estados poderosos, según Rawls, les compete ayudar a los débiles o proscritos a cumplir los deberes de derechos humanos que tienen respecto a sus propios ciudadanos, pero no tienen deberes de justicia ni de reducir las desigualdades entre Estados, o aún deberes de asegurar un mínimo nivel de bienestar para los ciudadanos fuera de su propia nación (Dwyer, 2005). Más aún, la diferencia estructural entre las funciones que Rawls asigna a las partes en las posiciones originales doméstica e internacional está asociada a dos diferentes concepciones de justicia económica (Pogge, 2004).

Por otra parte, están los abordajes cosmopolitas, que son aquellos que desean trascender la división geopolítica que es inherente a las ciudadanías nacionales de los diferentes Estados y países soberanos. Se niegan a aceptar la identidad patriótica dictada por los Gobiernos nacionales y afirman su independencia como ciudadanos del mundo o del cosmos. Una teoría de la justicia cosmopolita identifica una serie de normas de justicia y después argumenta que son universalmente aplicables sin tomar en cuenta factores como la ciudadanía común. Los cosmopolitas difieren ellos mismos sobre si la mejor justificación yace en un llamado a una humanidad común o a unas relaciones globales que desencadenan robustas normas sociales de justicia.

Algunos abordajes cosmopolitas son de corte utilitarista; otros están relacionados con la obligación de no dañar a través del respeto universal a los derechos humanos básicos, y los hay con carácter más inclusivo y contextual poniendo a la justicia global en estrecho vínculo con la definición de los distintos agentes globales y sus respectivas capacidades, posibilidades y límites de transformación. A continuación se hará una breve exposición de autores representativos de la postura cosmopolita.

\section{Postura utilitarista de la justicia global: Peter Singer}

Dentro de la bioética utilitarista existe un planteamiento muy conocido por su tendencia liberal en áreas como la eugenesia o el final de la vida, pero no de menor importancia en asuntos de justicia global. En relación con este último, se argumenta que el futuro en la era de la globalización dependerá de cómo se responda éticamente a la idea de que se vive en un único mundo. Si siempre ha sido una falta ética de las naciones poderosas no haber adoptado un punto de vista ético global, ahora también es, a largo plazo, un peligro para su seguridad (Singer, 2003).

Las fuerzas de mercado globales proporcionan incentivos para que cada nación se ponga lo que se ha llamado una camisa de fuerza dorada, es decir, un conjunto de políticas que implican liberalizar el sector privado de la economía, disminuir la burocracia, mantener baja la inflación y eliminar restricciones a la inversión externa (Freedman, 2000). Si un país 
se niega a ponerse la camisa dorada, la manada electrónica podría desbandarse en diferentes direcciones, y llevarse consigo las inversiones de capital que los países desean para mantener el crecimiento de sus economías. Y cuando ni el Gobierno ni los partidos políticos están preparados para asumir el riesgo de quitarse la camisa dorada, las diferencias entre los principales partidos se reducen a diferencias menores acerca de cómo ajustarse la camisa de fuerza. ${ }^{3}$

Singer (2003) —desde la reflexión sobre la contaminación atmosférica global— se pregunta qué es una distribución equitativa. En filosofía política es común seguir la distinción entre principios históricos y principios de tiempo concreto. Los primeros dicen que no se puede decidir si una distribución de bienes es justa o injusta sin tomar en cuenta la historia de cómo se llegó a la situación actual. ¿Tienen derecho las partes, por medio de una adquisición justificable en su origen y una cadena de transferencias legítimas, a las propiedades que ahora poseen? Si no lo tienen, se requiere una rectificación o compensación para producir una distribución justa. Los principios de tiempo concreto se fijan en la distribución existente en un mo-

3 Valga el ejemplo de lo que ha sucedido en México a partir de la apertura indiscriminada al libre comercio para confirmar los planteamientos de Singer. Con la industria farmacéutica sucede lo mismo: busca los mejores nichos mundiales para realizar sus inversiones y si no se está dispuesto a sus condiciones, migra a otros sitios. mento particular y cuestionan si esa distribución satisface algunos principios de justicia.

Los anteriores principios a la luz del principio de la mayor felicidad, propio de los utilitaristas, dirían lo siguiente: observar los principios históricos y de tiempo concreto redunda en un beneficio general. Aunque el principio igualitario no lo escogerían los utilitaristas, en ausencia de otro criterio claro para asignar porcentajes, puede ser un compromiso ideal que lleve a soluciones pacíficas, más que a un conflicto endémico. En la práctica, los utilitaristas podrían apoyar el principio de distribución de recursos a aquellos que están peor, porque cuando se cuenta con mucho, proporcionarle más a quien abunda no incrementa su utilidad, tanto como en el caso de que se tuviera poco (Singer, 2003).

En oposición a los nacionalistas, Singer argumenta que existe una sola comunidad de la que se debe ser corresponsable. Aunque los lazos de parentesco o amistad o incluso patrióticos son los naturalmente prioritarios, ies una razón suficiente para preferir ayudar a los conciudadanos que no tienen tanta necesidad frente a los extranjeros que tienen necesidades urgentes?

La concepción del nacionalismo de Anderson es un análisis de cómo la idea de pertenencia a una nación se mantiene en el mundo moderno. Considera la nación como una comunidad política imaginada, que vive solo en las mentes de aquellos que se ven a sí 
mismos como ciudadanos de la misma nación (Anderson, 1993). Para Singer, en la globalización es necesario preguntarse si, a largo plazo, será mejor continuar viviendo en las comunidades imaginadas llamadas Estados-nación, o empezar a considerarse todos como miembros de una comunidad imaginada mundial. Los problemas están en la actualidad demasiado interconectados como para poder ser resueltos adecuadamente por un sistema formado por Estados-nación.

La cuestión de si buscar una mayor igualdad dentro de las sociedades o entre las sociedades solo se plantea si no se pueden hacer ambas cosas; pero en ocasiones sí es posible. En la actualidad priman las obligaciones hacia los extranjeros frente a los conciudadanos, puesto que es más urgente reducir la pobreza absoluta de millones de seres humanos que disminuir la pobreza relativa de otros más cercanos. Se podría proponer como política pública que todo aquel que tenga suficiente dinero para gastar en lujos y frivolidades debería entregar a aquellos que tienen problemas para obtener lo suficiente para comer, que no tienen agua potable, ni cobijo, ni atención sanitaria, al menos el 1 \% de cada dólar de sus ingresos. Los que no cumplan con este criterio faltan a su parte justa de responsabilidad global y están haciendo algo moralmente reprobable (Singer, 2003).

En el campo de la bioética aplicada a la investigación internacional podría decirse que si esta pretende realizarse con criterios de justicia utilitarista, el principio del mayor bienestar para el mayor número tendría que aplicarse a nivel transnacional. Los enormes dividendos obtenidos por la industria farmacéutica al realizar sus investigaciones en países de mediano o bajo ingreso tendrían que considerar una distribución de beneficios menos unilateral y más acorde a las necesidades de los países huéspedes, dada la gran disparidad existente en su poder adquisitivo.

\section{Teoría cosmopolita de justicia global de Thomas Pogge}

Cambiando ahora de perspectiva, la reflexión se dirige a la justicia global basada en los derechos humanos. Desde este enfoque, se afirma que una concepción de justicia social es universalista, si y solo si:

- Impone a todas las personas el mismo sistema de principios morales fundamentales.

- Estos principios asignan los mismos beneficios morales fundamentales y las mismas cargas a todos.

- Estos beneficios y cargas fundamentales se formulan de modo que no se privilegie ni se perjudique de manera arbitraria a personas o grupos determinados (Pogge, 2005a).

El universalismo moral no puede definirse de manera formal o abstracta, por lo que ha de explicarse recurriendo a una aplicación ejemplar: el caso de la justicia 
económica. Tampoco es una posición moral con contenido material, sino un mero enfoque que hay que rellenar de tal manera que arroje posiciones morales sustantivas. Estas condiciones equivalen a una llamada a la coherencia sistemática en moralidad: la valoración moral de las personas y de sus conductas, de las reglas sociales y de los estados de cosas debe basarse en principios fundamentales que rijan para todas las personas por igual; además, cualesquiera discriminaciones construidas dentro de estos principios fundamentales deben recibir un fundamento plausible.

La preocupación por los problemas locales no debe impedir cobrar conciencia de lo que pasa a nivel global. Los ciudadanos de los países de alto ingreso son de algún modo responsables de la situación de pobreza a nivel mundial, pues no exigen a sus representantes que implementen políticas que ayuden a combatir la pobreza en los acuerdos que signan con los países de bajo ingreso.

El universalismo moral exige que se aplique una justificación plausible a la discordancia entre lo nacional y lo global respecto a la justicia mínima. Pogge tiene la impresión de que la mayoría de las personas de los países de alto ingreso piensa que el orden económico global es justo, aunque este no satisface dos requisitos mínimos que se exigen de cualquier orden económico nacional: las reglas sociales pueden ser modificadas pacíficamente por una amplia mayoría de aquellos a quienes se les imponen y toda pobreza evitable que amenace la vida debe eliminarse (Pogge, 2005b).

La valoración moral de un orden económico global debe ser sensible a la información de tres factores: la intensidad de pobreza absoluta, el grado de desigualdad que es una medida aproximada de lo evitable de la pobreza y del costo de oportunidad que conllevaría para los privilegiados evitarla, y la tendencia de los dos primeros factores, es decir, de qué modo tienden a evolucionar la pobreza y la desigualdad con el tiempo. Sobra decir que los tres factores los reprueba el orden económico global.

La inequidad en la distribución de la riqueza en el mundo no puede explicarse solo por causas locales, sino que también cuentan mucho los factores globales. Gracias a las racionalizaciones de los economistas, la mayoría de los habitantes del mundo rico cree que la pobreza extrema y su persistencia se deben exclusivamente a causas locales. Pocos resaltan que la pobreza severa es un daño que infligen los países ricos sobre los pobres globales. Muchos piensan que ayudar a los pobres es motivo de caridad, o que no hacerlo no los hace moralmente responsables de la privación continuada que sufren (Pogge, 2005b).

En definitiva, el Pogge plantea una aguda y dura crítica a la justicia global tal y como es proclamada y defendida por los países de alto ingreso. Demuestra las inconsistencias justificativas y propone una serie de medidas que podrían contravenir la injusticia 
inferida a millones de seres humanos en el planeta, sin afectar el bienestar de los países ricos.

\section{Teoría de justicia global según Onora O'Neill}

La globalización, en la que las fronteras de los Estados se han borrado, plantea el reto de repensar los principios de justicia internacional. Si antes los Estados-nación eran los agentes primarios de justicia, sin dejar de tener un papel importante, hoy se asiste a una nueva serie de relaciones internacionales. La justicia global se refiere a aquella que se establece donde existen relaciones o actividades no solo entre naciones, sino también entre corporativos, agencias gubernamentales, negociantes, comunidades, organizaciones caritativas, etc.

Cualquier discusión sobre justicia económica internacional debe tomar en cuenta la diversidad de capacidades y la posibilidad de acción de los varios agentes, así como las posibilidades y los límites de su transformación. Con base en la noción de capacidades de Amartya Sen (2009) puede determinarse qué obligaciones de justicia se pueden sostener y satisfacer y cuáles no. El mérito de enfocarse en capacidades es que presenta una preocupación explícita con la acción y con los resultados que los agentes pueden lograr en las circunstancias actuales, y así provee un serio punto de partida realista para un razonamiento normativo (O’ Neill, 2000). La otra consideración que necesita también tenerse en cuenta es el concepto de necesidades humanas de manera integral. Los seres humanos necesitan comida, vestido, higiene, cuidados médicos, sin los cuales enfermarán y morirán prematuramente. Dichas necesidades básicas proveerán una base para argumentar a favor de derechos básicos. Las necesidades humanas dan cuenta de los requerimientos reales del ser humano para vivir una vida buena o floreciente, la cual será difícil de lograr sin establecer una cantidad objetiva del monto de bien que necesita un ser humano.

Las capacidades para actuar libremente son tan fácilmente minadas, sea por pobreza, debilidad, inseguridad o dependencia, que el solo respeto del derecho a la libertad difícilmente alcanzará una realización amplia y efectiva de la justicia kantiana. Donde los agentes son vulnerables, la justicia sóoo puede encontrarse por una doble estrategia de disciplina de la acción del poderoso y de empoderamiento del débil, lo que hace de este alguien menos vulnerable (O’ Neill, 2000b).

En de los países de mediano o bajo ingreso, las transacciones económicas se hacen desde espacios lejanos y desregulados. Estas sociedades entran a la relación entre agentes con enormes disparidades en poder y recursos. Los débiles pueden sufrir por parte de quienes toman ventaja de su ignorancia y vulnerabilidad, debido a que nada les protege de las consecuencias no intencionadas de agentes y fuerzas económicas distantes. 
A nivel internacional, las diferencias de poder son abismales y es necesaria alguna estructura que limite el abuso o la explotación de la vulnerabilidad que se ejerce por parte de los poderosos. Más aún, el cambio institucional a larga escala pide también transacciones justas, y no solo desde lo externo, como es el respetar las formalidades de un contrato, préstamo o negociación. También requiere que no haya consentimiento espurio de los acuerdos o aceptación fácilmente obtenida por el vulnerable. Si se pretende no coercionar o explotar, no debe ejercerse presión indebida o irresistible sobre el vulnerable. ${ }^{4}$

La dependencia institucional hace muy difícil o imposible el disenso. A mayor dependencia estructural en las relaciones, los débiles más tienen que depender de la confianza respecto a que los fuertes no ejercerán las ventajas que su estatus superior les da. Puede que no exista injusticia como aducen los nacionalistas, pero si las instituciones dependen mucho de que los fuertes se abstengan de abusar sobre los débiles, no pueden confiablemente prevenir la injusticia. Mientras la búsqueda de los fuertes (económica o sexual) dependa de la ignorancia o aislamiento de los débiles para engañarlos, de sus oportunidades reducidas para actuar libremente, o de sus hábitos de deferencia y sosiego que son como la segunda naturaleza de los

$4 \quad$ Este tema tiene mucho que ver con el consentimiento informado otorgado en situaciones de vulnerabilidad social. débiles, aquellos cometerán injusticia (O' Neill, 2000b).

Existe una pluralidad de agentes de justicia que pueden jugar un papel en la institucionalización de los principios cosmopolitas de justicia. En las sociedades modernas, los agentes primarios de justicia han sido los Estados. Sin embargo, estos últimos a veces usan el poder no para conseguir la justicia sino para otros fines, que cuando son demasiado injustos, se podrían llamar Estados bribones. En otros casos los Estados fallan porque son muy débiles para actuar como agentes primarios de justicia, puesto que aunque llevan el título de Estados soberanos, en la práctica son dependientes o cuasiestados.

Los Estados bribones y poderosos confrontan a todos los agentes de justicia con grandes problemas. Contribuir a sus requerimientos contribuye a la injusticia, y tomar distancia ocasiona peligro y destrucción. Esto ha dado origen al tema de la justificación de la revolución y la resistencia o de la objeción contra tales requerimientos. En el caso de Estados dependientes queda indeterminado qué requiere la ley y los costos de cumplirla son grandes, al menos porque otros ni siquiera intentan hacerlo. Donde los agentes no pueden confiar en una ley imparcial, pueden encontrar en la corrupción y el nepotismo, en la compra de protección y de favores, y en la farsa sobre los requerimientos de justicia la manera de sacar adelante sus negocios. 
En el caso de Estados débiles, el enfoque desde las capacidades deja ver sentidamente por qué es importante pensar en otros posibles agentes de justicia. Los Estados débiles pueden solo carecer de recursos humanos, materiales y organizacionales para asegurar o mejorar la justicia dentro de sus fronteras; o adolecer de la capacidad para regular o influir la acción de ciertos agentes, para afectar aquello que ocurre en ciertas partes del Estado, o para alcanzar mayor justicia tal y como es el otorgar el bienestar necesario a su población. Pueden no representar los intereses de sus ciudadanos en foros internacionales y acordar en el extranjero tratados o préstamos insoportables; o carecer de la capacidad para prevenir o sofocar rebeliones y formas de feudalismo, insurgencia y secesión, bandidaje y carencia de estado de derecho, o para recaudar impuestos y hacer imperar la ley frente a poderosas mafias o grupos corruptos. Se habla a menudo de tales entidades como estados, pero se usa el término de manera honorífica, puesto que se conoce ampliamente su carencia de capacidades indispensables para cualquier agente de justicia.

A veces la carencia de capacidades es ocasionada porque otros agentes dentro o fuera del Estado-nación las han usurpado. Es el caso del narcotráfico en Colombia o México, o la fuerza militar adquirida por grupos secesionistas o insurgentes en África. El hecho de haber perdido el Estado tales capacidades no implica que otro agente las tomará a su cargo, o una simple ausencia general de poder, sino que genera una dispersión de capacidades entre un Estado débil y todos aquellos agentes de justicia activos dentro y alrededor de él, incapaces de asegurar mayor justicia. Peor aún, es posible que los Estados débiles puedan conservar capacidad de injusticia cuando son totalmente incapaces de hacer avanzar la justicia. En tales circunstancias otros logran ser importantes agentes de justicia.

Es necesario considerar también a los actores no-estado como agentes de justicia de frente a estados débiles como el mexicano. En relaciones internacionales, actores no-estado se refiere a ciertos tipos de agentes en cuanto ellos no son. No son ni el Estado, ni la creación de este, ni internacionales en el sentido de ser interestatales o intergubernamentales. Por ejemplo, las organizaciones no gubernamentales que operan a través de las fronteras, corporaciones transnacionales o multinacionales, y numerosos movimientos transnacionales que operan a través de las fronteras, tales como los movimientos sociales globales.

La distinción clara entre agentes primarios y secundarios de justicia solo puede darse donde existen Estados fuertes y relativamente justos, que regulan con éxito otros agentes dentro de sus fronteras. Pero en Estados débiles, la justicia ha de ser construida a partir de una diversidad de agentes con un amplio rango de capacidades, y que contribuyen a la justicia de maneras 
distintas a las generalmente conocidas (O’ Neill, 2000a). Este último sería el caso de México, lo cual se demuestra cada vez que se perciben signos de una democracia sumamente endeble, un estado de derecho muy vulnerado, la existencia de poderes fácticos, e incluso las acciones criminales de actores como el narcotráfico y el crimen organizado que han permeado a las instituciones garantes de seguridad, haciendo ostentación de poder.

Sin duda una reflexión bioética que parta de la acción al basarse en las capacidades, necesidades y posibilidades de acción de los distintos agentes, en las dependencias estructurales existentes en las relaciones comerciales, y en la configuración de los Estados en cuanto a su incapacidad para ejercer la justicia, tiene mucho que aportar al debate sobre lo justo en la investigación internacional. Aborda aspectos estructurales olvidados en otros enfoques de justicia y necesarios para entender la gran desigualdad entre los participantes en las investigaciones internacionales y saca a la luz la facilidad con que el fuerte puede abusar del débil partiendo de las capacidades y posibilidades concretas actuantes en cada situación.

\section{Teoría de justicia global de Powers y Faden}

Dentro de las teorías de la justicia global que parten de los contextos, o donde está la acción, el abordaje desde la justicia social es del todo relevante. Tiene que ver con aquellos contextos o determinantes sociales de la salud que limitan el bienestar de los individuos, lo que afecta varias de sus dimensiones y lo hace de manera duradera y profunda (Powers y Faden, 2006). La teoría de la justicia global se focaliza en la realización de múltiples dimensiones del bienestar a nivel internacional y en la identificación de los principales impedimentos para lograr este fin (Powers, 2013).

La teoría tiene dos objetivos. El primero se refiere a los acuerdos sociales justos, que deberían, en la medida de lo posible, asegurar un límite mínimo para cada uno de los seis elementos de bienestar necesarios para una vida humana decente: salud, conocimiento y comprensión, seguridad personal ante daño físico, psicológico y la amenaza de ambos; la posibilidad de asociación personal, el reconocimiento de grupos e individuos como merecedores de igual respeto y la capacidad de tener una vida autosuficiente. El segundo objetivo dice que los acuerdos sociales deberían combatir importantes formas de desventaja que crean y mantengan la injusticia que sufren las mayorías. Estas últimas son aquellas que necesitan trabajar mucho más duro o tener mucha suerte para lograr la vida decente de los que están socialmente posicionados con menor esfuerzo.

El bienestar lo describen Powers y Faden como una condición valuable eudemonísticamente, o buena para algunos y mala para aquellos que experimentan la carencia bajo el umbral 
requerido para una vida humana decente. La ventaja se refiere a estar en una posición superior con la facilidad que esto otorga para conseguir algo, frente a la desventaja del que está en una posición inferior.

Desde el punto de vista normativo, el objetivo primero de la justicia, relacionado con la distinción entre bienestar/ carencia, combate ciertas carencias de bienestar; el segundo objetivo de la justicia, relacionado con la ventaja/ desventaja, ataca la injusticia estructural de acuerdos sociales que distribuyen ventajas y desventajas de una manera particular.

Desde el punto de vista empírico, la carencia tiende a enmascararse dentro de la desventaja, y esta suele engendrar la carencia. Esta teoría se propone dejar ver los caminos por los que la carencia y la desventaja tienden a ir mano a mano, así como las diferencias conceptuales y normativas entre la carencia y la desventaja. Las carencias señaladas por el objetivo primero tienen que ver con las de las teorías de los derechos humanos, y las desventajas señaladas por el segundo se relacionan con las injusticias estructurales mostradas por las teorías igualitarias o revolucionarias. Al juntar ambos objetivos, esta teoría señala no solo las injusticias por falta de bienestar, sino aquellas injusticias que impiden sistemáticamente acceder a mínimos de bienestar, y los intrincados mecanismos por los que ambos niveles se retroalimentan y se superponen. Por ejemplo, además de señalar la falta de salud en una determinada población, muestra la carencia de atención de la salud del lugar, los pobres salarios para alimentarse sanamente y las jornadas excesivas de trabajo para sobrevivir.

El objetivo primero combate serias carencias producidas por varios determinantes más allá de los procesos sociales desventajosos desaprobados por el objetivo segundo. Reprueba, además, la falla de prevención o atención de carencias de bienestar debidas a desastres naturales, ataques terroristas o mala suerte. Esto es debido a que los reclamos que hacen los individuos de algún nivel de bienestar dependen mucho de la estructura organizada de la vida social. El objetivo segundo tiene mucho que ver con la justicia en curso o prospectiva en las relaciones sociales y con las tareas para remediar los duros efectos en el bienestar no hecho debido a patrones pasados de desventaja sistemática, todo ello por causa de acuerdos que distribuyen las ventajas y desventajas de manera sistemática, que favorecen a unos a expensas de un gran costo para otros.

Ambos objetivos se necesitan para no quedarse con una visión limitada de dimensiones del bienestar que no tome en cuenta el trasfondo estructural causal de las carencias, así como para definir el mínimo de bienestar requerido por la influencia negativa de los determinantes estructurales. Ningún objetivo por separado basta para una teoría de acuerdos sociales justos. Cada uno identifica una preocupación normativamente distinta de justicia, y cada uno está causalmente 
ligado con el éxito en el otro. Ambos objetivos combaten inequidades de varios tipos, pero de diferentes maneras y por diferentes razones frecuentemente superpuestas.

La resolución completa y duradera de estos problemas necesita reformas estructurales básicas del orden global, incluyendo reglas globales de comercio y prácticas internacionales de desarrollo. Esta teoría ofrece una justificación a la crítica de los patrones densamente tejidos de dominación y control político y económico internacional ejercido por los países de alto ingreso y las corporaciones multinacionales, sobre las naciones de mediano o bajo ingreso; también justifica la crítica a las variadas normas domésticas y culturales, a veces respaldadas por la fuerza de la ley, que contribuyen también a la opresión sistemática y a la consecuente disminución en varios elementos centrales del bienestar (Powers y Faden, 2013).

Este abordaje de justicia global es muy completo, pues articula ambos objetivos de la justicia, llamando a la complementación entre enfoques y a una acción desde distintos frentes, de tal manera que no solo se combata la carencia de bienestar puntualmente, sino que se eviten las causas que llevan a una constante y duradera deprivación de bienestar. La investigación internacional no está orientada a generar nuevos conocimientos y dispositivos para aliviar las razones de fondo por las cuales se enferman las poblaciones de los países de bajo ingreso, las llamadas "causas detrás de las causas". La reflexión bioética sobre lo justo necesita incorporar estos enfoques para hacer aportes más integrales.

\section{Enfoque de las capacidades en salud de Jennifer Ruger}

Se trata de una visión centrada en la agencia, entendida como aquella capaz de apoyar la participación directa de los individuos en acciones sociales, políticas y económicas, y habilitar para la toma de decisiones como sujetos activos del cambio. Además, promueve la capacidad de estos para entender y delinear su propio destino y ayudar a otros. Desde la perspectiva de las capacidades, el ejercer la libertad personal y participar en las opciones sociales debería moldear e influir en las políticas e instituciones. La política pública es un proceso, basado en valores y reglas, que expande la libertad individual.

Otro punto por considerar es la relación entre salud y agencia: a medida que exista salud, habrá capacidad de participar en la toma de decisiones dentro del sector salud y fuera de este. Al contrario, una agencia impedida, por opresión abierta o enmascarada, ocasiona un funcionamiento físico o mental impedido (Ruger, 2006).

Esta teoría sienta sus premisas en el principio de florecimiento humano que debe soportar la obligación universal de mantener y mejorar las capacidades en salud. El principio fundamenta el deber 
universal de reducir las desigualdades en las capacidades centrales en salud. Estas últimas se entienden como la capacidad y la libertad para lograr ciertos funcionamientos en salud, como por ejemplo librarse de la morbilidad y mortalidad prevenibles. Reducir las desigualdades en la salud de un individuo o comunidad tiene que ver con disminuir la brecha en el estatus actual de salud respecto al nivel óptimo.

Este enfoque contempla una gobernanza compartida en la que los individuos, los bienes en salud, los proveedores de servicios y las instituciones de salud trabajen juntos para reducir las diferencias. Para lograrlo, se requiere una amplia base de evidencia sobre la efectividad de los servicios de salud pública, la atención de la salud y las redes de soporte, y los determinantes sociales de la salud en cada país a lo largo del mundo (Ruger, 2010).

El anterior enfoque es diferente al de Daniels (2006), que aboga por políticas dirigidas a la igualdad de oportunidades, tales como invertir en educación básica, vivienda saludable, empleo seguro, etc. El paradigma de las capacidades necesita evaluar la salud y las políticas en salud en un contexto más amplio que requiere una comprensión de la justicia social. La salud y sus determinantes deben ser sopesados frente a otros fines sociales en una discusión democrática amplia en relación con los medios y fines de las políticas en salud. Dentro de estas, la atención de la salud es central, puesto que se depende de ella para que exista la salud necesaria para participar.

Tomando cierta distancia de las teorías cosmopolitas, el paradigma de Ruger no fundamenta una obligación general entre actores e instituciones en todo el mundo; más bien identifica dos principios para distribuir deberes específicos entre instituciones y actores: el compromiso voluntario y los requerimientos funcionales. El primero se refiere a aquel que voluntariamente se asume compartiendo recursos y renunciando a cierta autonomía a través de la acción colectiva para afrontar problemas en salud. Los requerimientos funcionales demandan que las instituciones y los actores tengan roles, capacidades y recursos necesarios para cumplir deberes específicos para remediar desigualdades globales en salud. Dichos deberes se distribuyen entre instituciones o actores, porque las funciones que asumen los hacen capaces de acometer los deberes.

La responsabilidad primaria de reducir desigualdades en salud la tienen los Estados, que regularmente son débiles. Los actores e instituciones globales tienen un papel secundario, de apoyo y facilitación para que los esfuerzos de los Estados en mejorar la salud de sus ciudadanos tengan éxito. Dichos actores e instituciones incluyen gobiernos, instituciones internacionales, ONG, fundaciones, etc.

Algunos deberes específicos para los actores involucrados serían generar y diseminar conocimiento e informa- 
ción a través de la creación de nuevas intervenciones en salud, adaptar las intervenciones en salud existentes y fortalecer la capacidad en investigación clínica. La investigación ha de favorecer el indagar en aquellas necesidades que son responsables de crear la diferencia entre el nivel ideal en salud y el actual. También el desarrollar intervenciones y servicios costo-efectivos para combatir las entidades que llevan a disminuir la mortalidad y morbilidad prematura, y los factores de riesgo que más contribuyen a la vulnerabilidad de las personas (Ruger, 2009).

\section{Balance y aplicación}

Después de haber presentado de manera sucinta algunos prototipos del debate sobre justicia global, se rescatarán algunos elementos de las aproximaciones cosmopolitas a lo justo en clave valorativa, y después se señalarán algunos caminos para acercarse a lo justo en la investigación biomédica internacional.

El utilitarismo global propuesto por Singer (2003) es un aporte importante al debate, principalmente por su sensible punto de partida ante la pobreza de las mayorías y el orden injusto de la humanidad. Al ser una doctrina consecuencialista, está muy atenta a las circunstancias, que en lo tocante a la distribución de bienes son desfavorables para la inmensa mayoría del planeta. Por otra parte, atender a la máxima beneficente es algo de por sí muy atractivo y necesario, lo cual hace cercana su propuesta teórica.

Su principal deficiencia es que como tal se trata del deber de ayudar puesto en práctica en base a la necesidad. La única razón de equidad que ofrece Singer es la igualdad de todas las personas como potenciales recipientes de ayuda de otros cuando están en necesidad. En general, no se mete con las razones por las que algunos pueden reclamar sus derechos o por las que los pobres son pobres y los ricos son ricos, y si esto último tiene que ver con la legitimidad del reclamo de sus derechos (Powers, 2013b). También es criticable la necesidad de ir más allá de la beneficencia para entrar en bases más robustas de la justicia.

No obstante las críticas, es necesario reconocerle su certero análisis de la situación mundial, su crítica a los postulados liberales y su propuesta alternativa de acción basada en la beneficencia y concretada en planteamientos plausibles, que cambiarían la suerte de mucha gente en este mundo.

La visión de Thomas Pogge es un aporte lúcido y crítico al debate actual sobre la justicia global. Su punto de partida realista, compartido por otros acercamientos, es incuestionable por el modo como confronta a los ciudadanos e instituciones de países de alto ingreso en su responsabilidad ante las desgracias de los pobres. El universalismo moral que propone está basado en la aplicación a todos del mismo sistema de principios morales fundamentales, con lo cual se 
evita un doble rasero para juzgar las causas de la pobreza global.

Pogge (2005b) se enfoca en los deberes negativos en el cumplimiento de los derechos humanos por parte de instituciones o países cuya ausencia amenaza contra la vida de muchas personas. Frente a las injusticias cometidas por los países de alto ingreso, propone una serie de medidas que podrían contravenir la injusticia hacia los pobres, sin afectar el bienestar de los primeros y ofreciendo varios cauces de acción al alcance de la mano. Su aporte tiene que ver con los derechos humanos negativos o de primera generación, los que enuncian el deber de no hacer daño. A diferencia de Rawls cuyo primer principio de justicia en su teoría de la justicia está basado en la libertad, Pogge sienta el derecho a no dañar en la pobreza concreta infringida a los menesterosos de la tierra, defendiendo los intereses de ellos y no un ideal justificador del status quo mundial.

La aproximación de Pogge es minimalista, porque se concentra en la obligación internacional de justicia para evitar dañar a la gente y causar déficits en la satisfacción de sus derechos humanos. Es minimalista por cuanto las personas están de acuerdo con los deberes negativos de no dañar aunque estén en desacuerdo con los deberes positivos de ayudar (Daniels, 2006). No obstante, es valiosa la propuesta de Pogge puesto que establece una valoración moral contundente del orden establecido y caminos concretos para la reversión del problema de la pobreza mundial.
Onora O'Neill (2000b) ofrece un punto de partida práctico, donde está la acción y se enfoca en las necesidades integrales de los agentes, en sus capacidades y posibilidades, asumiendo realistamente las posibilidades y los límites de transformación. Su acercamiento teórico es mucho más cercano a los problemas de justicia global, puesto que al asumir un punto de partida práctico, evita de entrada las justificaciones del statu quo en nombre de la libertad o del Estado-nación, piezas centrales de un sistema económico individualista y consumista.

Al adentrarse en las necesidades humanas de manera integral, permite considerar al ser humano como persona con una determinada calidad de vida, en la cual han de incluirse elementos de su mundo subjetivo, relacional e incluso trascendente, que le hagan florecer. Al tomar en cuenta para la justicia internacional el mundo de las relaciones internacionales de manera empírica, con sus rejuegos de dominación y poder, permite definir con más claridad qué obligaciones de justicia se pueden sostener y cuáles no. Y al definir a los agentes primarios y secundarios de justicia, O’Neill ofrece una descripción realista de algunos Estados y el nivel de dependencia o alianzas perversas a las que pueden llegar, o la debilidad que les caracteriza al grado de poder ser calificados como ausentes de estado de derecho.

Finalmente, señala O' Neill (2000b) la importancia de hacer ver los abusos de los poderosos y la vulnerabilidad del 
débil en las negociaciones tan dispares de poder en el terreno de las relaciones internacionales, enmascaradas por unos acuerdos legales por el cumplimiento de contratos, pero lejanas a las necesidades y capacidades reales de los contratantes. De aquí se desprende la impostergable tarea de disciplinar al poderoso y empoderar al débil, de tal manera que los acuerdos sean más equitativos y realmente hagan justicia que redunde en beneficios compartidos equitativamente en la vida real.

Powers y Faden (2013), desde su original postura respecto a la justicia global, critican aquellos abordajes de justicia limitados al bienestar, enriqueciendo esta explicación con la de las desventajas sistemáticas a nivel social y a lo largo de la historia que impiden que grandes mayorías de la población logren satisfacer requerimientos mínimos de bienestar. Al igual que O'Neill desmienten el statu quo que justifica el orden mundial establecido con base en grandes ventajas para pocos y muchas desventajas para las mayorías, y consideran al ser humano en su desarrollo de manera integral, para evitar así reduccionismos de diversa índole que limitan al ser humano a su capacidad de consumo, o de ejercer su autonomía.

Señalan también Powers y Faden (2013) la necesidad de tomar en cuenta el reforzamiento que se dan entre sí los dos niveles, y crean así patrones entretejidos de injusticia muy difíciles de cambiar. En lugares donde existen tales patrones de injusticia sistemática, como es el caso de México, se explica más claramente por qué muchas personas, a menos que tengan muy buena suerte, no pueden dejar la pobreza o la falta de educación, puesto que no basta con querer salir de ello - lo cual pide un soporte motivacional y valorativo muchas veces ausentesino tener recursos estructurales que lo permitan, como un buen sistema educativo, adecuada alimentación, seguridad suficiente para tener una vida pacífica, salario digno para evitar jornadas dobles o triples de trabajo, servicios públicos de calidad para llevar una vida digna, etc. También explica que los pocos que salen de los círculos de pobreza y desventaja sistemática repiten los patrones de dominación y violencia, dado que no existe un sistema social que impida su reproducción al limitar al poderoso y empoderar al débil.

El punto de vista crítico es uno de los aportes centrales de Powers y Faden, que señalan cómo las injusticias estructurales son causa y efecto de relaciones internacionales injustas, dictadas por grupos de poder que gobiernan al mundo, sea a nivel político o económico. En el primer caso está la política mundial de corte intervencionista dictada en Estados Unidos, favorecedora de sus intereses geopolíticos como Israel; en el segundo, las normas del Banco Mundial o el Fondo Monetario Internacional, que

\footnotetext{
Lo anterior no quita la responsabilidad doméstica por parte de los gobiernos ante las carencias de bienestar que sufren las mayorías en las naciones empobrecidas.
} 
con sus reglas sumen en la dependencia a muchas economías nacionales. El nivel económico está también representado por las compañías multinacionales, como la industria farmacéutica, cuyo poder financiero es enorme y al extender sus negocios globalmente lo hacen de maneras muy inequitativas o ventajosas, $y$ de esta manera se queda la gran mayoría de las ganancias en pequeños sectores. ${ }^{5}$

El enfoque de Ruger basado en las capacidades y funcionamientos es sin duda un abordaje bastante atractivo, dirigido a empoderar a distintos actores o incluso instituciones que se comprometan en participar en espacios ciudadanos orientados a disminuir la brecha en desigualdades en salud, y no por ello liberar de su responsabilidad al Estado como garante primario de la atención de la salud. Asimimso, da pautas para participar en asuntos de salud pública relacionados con la disminución de la desigualdad en salud en sitios de estado débil, donde existen múltiples determinantes sociales de la salud; en el terreno de la investigación en salud, se dirige a fortalecer y capacitar para la investigación en las grandes necesidades en salud o en aspectos de farmacoeconomía, orientados a disminuir los indicadores de falta de acceso a la salud, como los de morbilidad o mortalidad infantil.

Podría criticarse de estas teorías centradas en la acción y en los contextos su ideología de corte social que subyace a sus planteamientos, lo cual sin duda es cierto. Sin embargo, la misma crítica puede hacerse a los otros enfoques nacionalistas, en el sentido de ser defensoras del liberalismo. No hay teoría que quede libre de elementos a priori no confesados.

A continuación se señalan algunas situaciones en las cuales se pueden aplicar los marcos reflexivos expuestos a la investigación internacional de nuevos medicamentos patrocinada por la industria farmacéutica. El enfoque de justicia global como el de O' Neill (2000) o el de Powers y Faden (2006) señala la importancia de tomar en cuenta los patrones sistemáticos de ventaja/desventaja. Uno de ellos es el cociente 10/90 que hace que los fondos destinados a investigar se concentren en una minoría. Investigar es desarrollar conocimiento y este es un medio central para generar riqueza, autodeterminación y soberanía. Si no se establecen medidas para revertir el cociente 10/90, como el hecho de que los beneficios de las investigaciones realizadas en países de bajo ingreso redunden en ayudas sociales que reviertan las grandes necesidades en salud, las investigaciones reforzarán el statu quo vigente (Declaración Universal sobre Bioética y Derechos Humanos, 2005, London, 2005, Páez Moreno, 2015).

Otras medidas que pueden emprenderse por parte de quienes buscan hacer investigación y evitar el conflicto de intereses son el hecho de sumarse a las pautas señaladas por el Health Impact Fund, que ha diseñado un mecanismo que ofrece a los innovadores farmacéuticos una recompensa económica a partir de un fondo común, basada en el impacto 
en la salud de sus productos para enfermedades esenciales, si están de acuerdo con venderlos al costo (Hollis y Pogge, 2008). De esta manera, se revertiría el interés dominante en las investigaciones internacionales: lucrar a expensas de descubrir medicamentos que tienen poco que ver con las grandes necesidades en salud de los países huéspedes.

Una tercera medida de justicia global estaría en la línea de revertir el sistema actual de patentes en caso de epidemias, concretado en los Acuerdos de Propiedad Intelectual (TRIPS) firmados por los países de la Organización Mundial de Comercio (омc), el cual estableció estándares de protección de patentes para los productos farmacéuticos. En 2001 la Organización Mundial de la Salud (oms) emitió la Declaración del TRIPS y salud pública, o Declaración de Doha, la cual establece que los acuerdos TRIPS han de interpretarse de manera que en los países de la omc se proteja el derecho a la salud pública y de manera especial el derecho a medicinas para todos, de donde se derivó la posibilidad de contraer licencias obligatorias o compulsory licenses. Estas son licencias acordadas entre el propietario de la patente y cierto país para determinados usos y modos de utilización, como los implementados por Sudáfrica, Brasil e India para la creación de antirretrovirales genéricos frente a la epidemia de SIDA. Las batallas se han ganado gracias a la postura de los Gobiernos locales y la presión de la opinión pública (Drahos y Braithwhaite, 2002).
Otra medida en la línea de limitar las seducciones de los fuertes, que en el terreno de la investigación internacional en seres humanos tienen que ver con el poder económico de la industria farmacéutica para rendir a sus intereses a las instituciones de salud y las autoridades locales, tiene que ver con la formación y capacitación de los comités de ética en investigación. Desde la justicia global, estos son una instancia fundamental de arbitraje, por ejemplo, para impedir aquellos estudios cuya pregunta de investigación sea poco útil para el contexto donde se investiga, o en las que los beneficios sean superfluos, sin mejorar de alguna manera las instituciones o las grandes necesidades en salud locales, comparados con la enorme ganancia económica que se lleva la industria farmacéutica. Sin los pronunciamientos de los comités de ética difícilmente habrá quien tenga la capacidad a título personal de impedir ofertas de investigación que reportan cierto beneficio local, ante la restricción presupuestaria para generar investigación nacional.

Finalmente, en un orden internacional injusto en el que los ciudadanos de países de alto ingreso se benefician de la investigación llevada a cabo en países de mediano o bajo ingreso, se plantea la obligatoriedad de remediar la situación de aquellos por quienes uno se ha beneficiado (Pogge, 2005b; Malmkvist, 2014), al gozar de los éxitos terapéuticos de nuevas moléculas investigadas en otras latitudes, muchas veces con pacientes en situación de vulnerabilidad 
que no podrán recibir dicho beneficio. En otras palabras, beneficiarse de la injusticia - de una situación que no debería existir- puede originar responsabilidades hacia las víctimas, aunque no se haya sido causante directo o haya un vínculo particular con estas últimas. La injusticia está en que se ha investigado aprovechándose de la necesidad de los pacientes pobres de reclutarse en protocolos para obtener atención médica. Se trata de un compromiso en el cual todos deberían incluirse a través de cualquier acción colectiva a favor de evitar la explotación de los pobres o de ayudar a su desarrollo, por ejemplo a través del Global Forum for Health Research (2015).

\section{Conclusiones}

La investigación internacional de nuevos medicamentos patrocinada por la industria farmacéutica es un fenómeno propio de la globalización. Como tal, está caracterizada por un conflicto de intereses que tiene muchas facetas, que confluyen en la dificultad o imposibilidad de conciliar la neutralidad científica y el deber de generar conocimiento y alternativas terapéuticas a favor de la humanidad, y el interés por generar ganancias desproporcionadas que obedecen a las dinámicas del mercado global.

Al ser un fenómeno internacional en el que confluyen actores con una gran desigualdad de poder económico y científico, necesita ser evaluado por una bioética global, particularmente desde el ángulo de la justicia. Sin embargo, no todos los abordajes ofrecen un punto de vista crítico sobre la realidad de opresión y abuso establecida, ni una reflexión bioética coherente con esta. En el debate bioético sobre lo justo en las investigaciones internacionales de nuevos medicamentos, así como sobre el reparto justo de beneficios a nivel global, es necesario un marco teórico alternativo de justicia global.

Las teorías nacionalistas, aunque son sensibles a la pobreza e injusticia mundial, no se salen de un esquema doméstico que les impide atravesar fronteras, y que en el fondo defiende sus privilegios e intereses, culpando a los otros de su estado de vulnerabilidad a la vez que se exime de su responsabilidad compartida. Las teorías cosmopolitas son mucho más atractivas para quienes viven en un contacto más directo con la pobreza de las mayorías, pero en su afán de universalizar caen fácilmente en idealismos que siempre quedarán inalcanzables para el quehacer ético. El abordaje de justicia global de Singer, sin duda del todo lúcido por el diagnóstico que establece, quizás caiga en esta categoría.

Dentro del enfoque cosmopolita, algunos teóricos ofrecen modalidades más específicas o más contextuales y particulares, aunque cada uno de diversa índole. El abordaje de Pogge sobre la necesidad de establecer mínimos comunes que respeten el derecho a no ser dañado o empobrecido es de suma importancia y se ha concretizado 
a través de iniciativas como el Health Impact Fund. Las teorías donde está la acción, como las de Onora O’Neill, Powers y Faden, o Jennifer Ruger, a partir de un abordaje de justicia global práctico y contextualizado y desde una perspectiva estructural, son indispensables en vistas a guiar acciones justas en la investigación biomédica internacional, al estar esta relacionada con una gran complejidad de actores sociales, económicos y políticos, y determinada por graves factores estructurales. Parten de las capacidades y necesidades concretas entre las partes, tienen como referencia el bienestar especificado en indicadores y están dirigidas a orientar acciones que incidan en recuperar la salud vista de manera integral.

A partir de los enfoques contextuales de justicia se pueden delinear diversas pautas para investigaciones internacionales, orientadas a solucionar las desigualdades de fondo, tales como una justa distribución de beneficios a favor de las instituciones y poblaciones huéspedes, el sumarse a iniciativas globales dirigidas a contrarrestar la injusticia o la formación de comités de bioética en investigación capacitados que puedan servir de filtro ante pesquisas que reprueben estándares de justicia social.

\section{Referencias}

Anderson, B. (1993). Comunidades imaginadas. México, D. F: Fondo de Cultura Económica.
Bekelman, J., Mphil, Y. y Gross, C. (2003). Scope and impact of financial conflicts of interest in biomedical research. JAMA, 289(4), 454-465.

Bollyky, T. (2013). Access to drugs for treatment of noncommunicable diseases. PLOS Medicine, 10(7). Recuperado de http://www.plosmedicine.org/article/ info\%3Adoi\%2F10.1371\%2Fjournal. pmed. 1001485.

Brooks, T. (2008). The global justice reader. Malden, Oxford, Victoria: Blackwell Publishing.

Daniels, N. (2006). Equity and population health. Toward a broader bioethics agenda. Hastings Center Report, 36(4), 22-35.

Declaración Universal sobre Bioética y Derechos Humanos (2005). Aprovechamiento compartido de los beneficio. Génova: Unesco.

Drahos, P. y Braithwaite, J. (2002). Informational feudalism. London, Sterling: Earthscan Publications.

Dwyer, J. (2005). Global health and justice. Bioeth, 19(5-6), 460-475.

Freedman, T. (2000). The Lexus and the olive tree. New York: Anchor Books.

Global Forum for Health Research (2015). Home page. New York: WHO. Recuperado de http://www.who.int/workforcealliance/members_partners/member_list/ gfhr/en/

Hollis, A. y Pogge, T. (2008). Health impact fund. Making new medicines accesible for all. Incentives for Global Health. Recuperado de http://healthimpactfund.org/wp-content/uploads/2015/12/ hif_book.pdf 
London, A. (2005). Justice and the development approach to international research. Hastings Center Report, 35(1), 24-37.

Macklin, R. (2004). Ética de la investigación internacional. Acta Bioethica, 10(1), 17-35.

Malmkvist, E. (2014). International clinical research and the problem of benefiting from injustice. Conference paper. Sweden: Linköping University. Recuperado de http:// liu.diva-portal.org/smash/record.jsf?pi$\mathrm{d}=\operatorname{diva2} \% 3 \mathrm{~A} 828353 \& \mathrm{dswid}=4938$

Michaud, C. y Murray, Ch. (1996). Resources for health research and development in 1992: a global overview. En Ad Hoc Committee on Health Research Relating to Future Intervention Options (Ed.), Investing in Health Research and Development (pp. 213-234). Géneva: World Health Organization.

Nagel, T. (2005). The problem of Global Justice. Philosophy and Public Affairs, 33(2), 113-147.

O'Neill, O. (2000a). Agents of Justice. En T. Pogge (Ed.), Global justice (pp. 188203). Oxford: Blackwell Publishing.

O'Neill, O. (2000b). Bounds of justice. New York: Cambridge University Press.

Páez Moreno, R. y García de Alba, J. (2009). International research and just sharing of benefits in Mexico. Develop World Bioethics, 9(2), 65-73.

Páez, R. (2011). La investigación de la industria farmacéutica: ¿condicionada por los intereses del mercado? Acta Bioethica, 17(2), 237-246.

Páez, R. (2015). Pautas bioéticas. La industria farmacéutica entre la ciencia y el mercado, México, D. F: Fondo de Cultura Económica, Universidad Nacional Autónoma de México [unam].

Pignarre, P. (2005). El gran secreto de la industria farmacéutica. Barcelona: Gedisa.

Pogge, T. (2002). Responsabilities for poverty related ill health. Ethics \& International Affairs, 16(2), 71-79.

Pogge, T. (2004). La incoherencia entre las teorías de la justicia de Rawls. Revista Internacional de Filosofía Politica, (23), 28-48.

Pogge, T. (2005a). World poverty and human rights. Symposium. Ethics EInternational Affairs, 19(1), 1-7.

Pogge, T. (2005b). La pobreza en el mundo y los derechos humanos. Barcelona: Paidós, Estado y Sociedad 134.

Powers, M. (2013). Global justice and human rights: a brief overview. Recuperado de http://www.fewresources.org/global-justice.html

Powers, M. y Faden, R. (2006). Social justice. The moral foundations of public health and health policy. New York: Oxford University Press.

Powers, M. y Faden, R. (2013). Social practices, public health and the two aims of justice: responses to comments. Public Health Ethics, 6(1), 45-49.

Rawls, J. (2001). El derecho de gentes y una revisión de la idea de razón pública. Barcelona: Paidos, Estado y Sociedad 86.

Ruger, J. (2006). Health, capability and justice toward a new paradigm in health, policy and law. Cornell Jorunal of Law and Public Policy, 15(2), 101-187.

Ruger, J. (2009). Global health justice. Public Health Ethics, 2(3), 261-275.

Ruger, J. (2010). Health and social justice. Oxford: Oxford University Press. 
Sen, A. (2009). The idea of justice. Cambridge: The Belknap Press of Harvard University Press.

Singer, P. (2003). Un solo mundo. La ética en la globalización. Barcelona: Paidós, Estado y Sociedad 113.

Vernaz, N., Haller, G., Girardin, F. et al. (2013). Patented drug extension strategies on healthcare spending: a cost-evaluation analysis. PLOS Me- dicine, 10(6). Recuperado de http:// www.plosmedicine.org/article/info\%3Adoi\%2F10.1371\%2Fjournal. pmed.1001460.

World Medical Association. (2015). WMA Medical Ethics Manual (3ra. ed.). Recuperado de http://www.wma.net/ es/30publications/30ethicsmanual/pdf/ ethics_manual_en.pdf 